\title{
Hodge-Riemann Relations for Polytopes A Geometric Approach
}

\author{
Gottfried Barthel, Jean-Paul Brasselet, \\ Karl-Heinz Fieseler, Ludger Kaup
}

\begin{abstract}
The key to the Hard Lefschetz Theorem for combinatorial intersection cohomology of polytopes is to prove the Hodge-Riemann bilinear relations. In these notes, we strive to present an easily accessible proof. The strategy essentially follows the original approach of $\underline{\mathrm{Ka}}$, applying induction à la $\mathrm{BreLu}_{2}$, but our guiding principle here is to emphasize the geometry behind the algebraic arguments by consequently stressing polytopes rather than fans endowed with a strictly convex conewise linear function. It is our belief that this approach makes the exposition more transparent since polytopes are more appealing to our geometric intuition than convex functions on a fan.
\end{abstract}

\section{Introduction}

The proof of the Hard Lefschetz Theorem for the "Combinatorial Intersection Cohomology" of polytopes given in $\mathrm{Ka}$ was the keystone in a long endeavour of several research groups to verify that Stanley's generalized ("toric") $h$-vector for polytopes has the conjectured properties: The theorem (usually referred to as "HLT" in the sequel) implies that the generalized $h$-vector agrees with the vector of even degree Intersection Cohomology Betti numbers and that this vector enjoys the unimodality property (in addition to symmetry and non-negativity).

The HLT is an easy consequence of the so-called bilinear "Hodge-Riemann relations" ("HR relations" or "HRR" for short); and since the latter, being a "positivity result", reflect convexity in a more appropriate way than the HLT, the focus has shifted towards proving these relations. The first proof of the HRR given in $\mathrm{Ka}$. has been rather involved. The task of making it more easily accessible has been taken up in different articles, cf. $\mathrm{BreLu}_{2}$ and $\mathrm{BBFK}_{3}$. With the present notes, we further pursue this direction: Being convinced that polytopes are closer to our geometric intuition, we present an approach that 
stresses geometric operations on polytopes rather than algebraic operations on strictly convex conewise linear functions.

Let us briefly recall the setup, referring to section 4 for further details: To an $n$ dimensional polytope $P$ in an $n$-dimensional real vector space $V$, one associates its outer normal fan $\Delta=\Delta(P)$ in the dual vector space $V^{*}$, and a conewise linear strictly convex function $\psi$. The "combinatorial intersection cohomology" $I H(\Delta)$ is a finite-dimensional real vector space with even grading $\bigoplus_{k=0}^{n} I H^{2 k}(\Delta)$. There is a perfect pairing

$$
\cap: I H^{q}(\Delta) \times I H^{2 n-q}(\Delta) \longrightarrow \mathbf{R}
$$

the "intersection product", so Poincaré duality holds on $I H(\Delta)$.

On $I H(\Delta)$, the multiplication with $\psi$ induces an endomorphism

$$
L: I H^{q}(\Delta) \longrightarrow I H^{q+2}(\Delta)
$$

called the Lefschetz operator. The key result of $[\mathrm{Ka}]$ (see also $\left[\mathrm{BreLu}_{2}\right)$ reads as follows: Hard Lefschetz Theorem (HLT). For each $k \geqq 0$, the iterated Lefschetz operator

$$
L^{k}: I H^{n-k}(\Delta) \longrightarrow I H^{n+k}(\Delta)
$$

is an isomorphism.

By Poincaré duality, it suffices to prove that each map $L^{k}$ be injective or surjective.

Using the intersection product, the Hard Lefschetz Theorem can be restated in a different framework: Each mapping $L^{k}$ (for $k \geqq 0$ ) yields a bilinear form

$$
s_{k}: I H^{n-k}(\Delta) \times I H^{n-k}(\Delta) \longrightarrow \mathbf{R}, \quad(\xi, \eta) \longmapsto \xi \cap L^{k}(\eta),
$$

called the $k$-th Hodge-Riemann bilinear form, or "HR-form" for short. This form is symmetric since $L$ is self-adjoint with respect to the intersection product. In this set-up, the HLT is equivalent to the non-degeneracy of all forms $s_{k}$.

Beyond non-degeneracy, the HR relations provide explicit formulæ for the signatures of these pairings. To that end, we have to consider the primitive intersection cohomology

$$
I P^{n-k}(\Delta):=\operatorname{ker}\left(L^{k+1}: I H^{n-k}(\Delta) \longrightarrow I H^{n+k+2}(\Delta)\right)
$$

for $0 \leqq k \leqq n($ with $k \equiv n \bmod 2)$. In fact, assuming the HLT, there is an $s_{k}$-orthogonal decomposition

$$
I H^{n-k}(\Delta)=L\left(I H^{n-k-2}(\Delta)\right) \oplus I P^{n-k}(\Delta)
$$

More generally, we see: 
Proposition 1.1. If the HLT holds for the Lefschetz operator $L$ on the intersection cohomology of the fan $\Delta=\Delta(P)$, then, for each $k$, the intersection cohomology splits as an orthogonal direct sum

$$
I H^{n-k}(\Delta)=\bigoplus_{j \geqq 0} L^{j}\left(I P^{n-k-2 j}(\Delta)\right) .
$$

Now for each $q \leqq n-2$, the restricted operator $L$ provides an isometric embedding $I H^{q}(\Delta) \hookrightarrow I H^{q+2}(\Delta)$ with respect to the pertinent HR-forms. Hence, in order to determine the signature of $s_{k}$, it suffices to consider the restrictions of the Hodge Riemann forms $s_{k+2 j}$ to the corresponding primitive subspaces $I P^{n-k-2 j}(\Delta)$. Here is the statement:

Hodge-Riemann Bilinear Relations (HRR). For each $k \geqq 0$ (with $k \equiv n \bmod 2$ ), the Hodge-Riemann bilinear form $s_{k}$ is $(-1)^{(n-k) / 2}$-definite on $I P^{n-k}(\Delta)$.

The HR relations imply the HLT, since the HR forms are readily seen to be nondegenerate by descending induction on $k$ : For $k=n$, that follows from $I P^{0}(\Delta)=I H^{0}(\Delta)$. For $k<n$, we assume that $s_{k+2}$ is non-degenerate. Then so is the restriction of $s_{k}$ to $L\left(I H^{n-k-2}(\Delta)\right)$. This implies

$$
I H^{n-k}(\Delta)=L\left(I H^{n-k-2}(\Delta)\right) \oplus L\left(I H^{n-k-2}(\Delta)\right)^{\perp},
$$

and it now suffices to prove $L\left(I H^{n-k-2}(\Delta)\right)^{\perp}=I P^{n-k}(\Delta)$. The inclusion " $\supset$ " follows from the fact that $L$ is $\cap$-self-adjoint, while " $\subset$ " is a consequence of Poincaré duality for the complementary dimensions $n-k-2$ and $n+k+2$.

From Proposition 1.1, we immediately obtain a reformulation of the HRR in which the primitive cohomology does not enter explicitly:

Proposition 1.2. The HRR are equivalent to the HLT together with the additional condition that the Hodge-Riemann bilinear forms $s_{k}$ on $I H^{n-k}(\Delta)$ satisfy the "HR-equation"

$$
\operatorname{sign}\left(s_{k}\right)=\operatorname{sign}\left(s_{k+2}\right)+(-1)^{(n-k) / 2}\left(b_{n-k}-b_{n-k-2}\right),
$$

where $b_{q}:=\operatorname{dim}_{\mathbf{R}} I H^{q}(\Delta)$ denotes the $q^{\text {th }}$ intersection cohomology Betti number of the fan $\Delta$.

\section{Outline of the proof of the Hodge-Riemann rela- tions}

The HR relations are known to hold if the polytope $P$ is simple. The first proof has been given in $[\mathrm{Mc}$; a simplified version followed in $\mathrm{Ti}$. This result is the basis for the 
proof of the general case by a twofold induction: The "outer loop" is on the dimension $n:=\operatorname{dim}(P)$. For the more involved "inner loop", following BreLu 2 , we associate to $P$ an integer $\mu:=\mu(P) \geqq 0$ that measures how far $P$ is from being simple: It counts those faces, here called "normally stout" (see [3.6), that witness non-simplicity, with $\mu=0$ characterizing simple polytopes. The inner induction on $\mu$ requires three main steps:

Cutting off (Section 3): Given a face $F \prec P$, we consider an affine hyperplane $H$ that is sufficiently near and parallel to a supporting hyperplane for the face $F \prec P$ and intersects $\stackrel{\circ}{P}$. Let $P=G \cup R$ be the corresponding decomposition of $P$ into the "germ $G=G_{P}(F)$ of $P$ along the face $F$ " and the residual polytope $R$.

Then, if $F \prec P$ is a normally stout face of minimal dimension, we have $\mu(R)<\mu(P)$, so the HRR hold for $R$ by induction hypothesis. For the investigation of the germ $G$, it is important that the face $F$ itself is a simple polytope and that it is "normally trivial" in $P$, cf. 3.12

HRR for special $\boldsymbol{n}$-polytopes (Section 5): Assuming the HRR to hold for $m$-polytopes (with $m<n$ ), we prove the validity for the following special $n$-polytopes:

5.1 A pyramid $P=\Pi(Q)$ with an $(n-1)$-dimensional base $Q$.

5.2 A non-trivial product $P=S \times P_{0}$, where $S$ is simple.

Furthermore, we prove the following Gluing property:

5.3 The HRR hold for an $n$-polytope $P$ that can be cut "transversally" into two polytopes $P_{1}$ and $P_{2}$ such that the HRR hold for both pieces.

Deformation of the germ $G$ into a product (Section [6): There is a continuous family $\left(Q_{t}\right)_{t \in[0,1]}$ of pairwise combinatorially equivalent polytopes with $Q_{1}=G$ and $Q_{0}=$ $F \times \Pi(L)$ with the pyramid $\Pi(L)$ over a "link" $L=L_{P}(F)$ of $F$ in $P$. Then HRR is valid for $Q_{1}=G$ iff it is for $Q_{0}$.

By induction hypothesis, the HRR hold for the lower dimensional polytopes $F$ and $L$, and thus, by 5.1 and 5.2, hold also for $Q_{0}=F \times \Pi(L)$, hence eventually also for $G$. Finally, the gluing result of 5.3 applied with $P_{1}=R$ and $P_{2}=G$ from Step 1 ("Cutting off") yields the HRR for the initial polytope $P$.

In section 4 , we recall the definition and basic properties of combinatorial intersection cohomology as needed later on. 


\section{Cutting off}

In this section, we explain how a polytope can be made simple by successively cutting off faces containing non-simple points. In that process, we have to make sure at each step that we get closer to the class of simple polytopes. A measure for the "distance" of a polytope $P$ to that class is the number $\mu(P)$ of its "normally stout" faces, see Def. 3.6

We first introduce some basic constructions.

Remark and Definition 3.1. The complement $V \backslash H$ of an affine hyperplane $H \subset V$ consists of two open connected components

$$
V \backslash H=U_{1} \cup U_{2} .
$$

We say that a subset $A \subset V$ lies strictly on one side of $H$ if either $A \subset U_{1}$ or $A \subset U_{2}$.

Let $P \subset V$ be a polytope, and $H$, a hyperplane as above. We call $H$ a cutting hyperplane for $P$ if it intersects the relative interior, i.e., $H \cap \stackrel{\circ}{P} \neq \emptyset$. Such a hyperplane yields a decomposition

$$
P=P_{1} \cup P_{2}
$$

of $P$ into polytopes $P_{i}:=P \cap \overline{U_{i}}$ with $\operatorname{dim} P_{1}=\operatorname{dim} P_{2}=\operatorname{dim} P$. Both pieces meet along the common facet $P_{1} \cap P_{2}=H \cap P$ which we call the cut facet.

We say that $H$ cuts $P$ transversally if no vertex of $P$ lies on $H$. Moreover, for a proper face $F \supsetneqq P$, we say that such a transversal hyperplane $H$ is sufficiently near to $F$ (or a "nearby hyperplane") if $F$ lies strictly on one side of $H$, whereas all the remaining vertices of $P$ (i.e., those not contained in $F$ ) lie on the other side. If in addition $H$ is parallel to a supportimg hyperplane $H_{0} \subset V$ for the face $F \prec P$, i.e. $P \cap H_{0}=F$, we also say that $H$ "cuts off" the face $F$.

In the sequel, cutting off a proper face $F$ of $P$ by a nearby parallel hyperplane plays an important role: The resulting decomposition

$$
P=G \cup R
$$

of $P$ into one polytope $G$ containing the face $F$ and a "residual polytope" $R$ allows a "divide et impera" approach to the HRR problem.

Definition 3.2. Let $F \supsetneqq P$ be a proper face of a polytope $P$ in $V$.

1. A germ $G=G_{P}(F)$ of $P$ along the face $F$ is any polytope $G$ obtained as follows: Choose an affine hyperplane $H_{0}$ in $V$ with $P \cap H_{0}=F$ and let $H$ be a parallel hyperplane such that $F$ lies strictly on one side, say $U_{1}$, of $H$, and the vertices of $P$ not contained in $F$ on the other side $U_{2}$. Then $G:=P_{1}$, while $R:=P_{2}$ is the corresponding residual polytope. 
2. A link $L=L_{P}(F)$ of the face $F \supsetneqq P$ is any polytope obtained in the following way:

- If $F=\{\mathbf{a}\}$, then $L_{P}(F):=L_{P}(\mathbf{a}):=P \cap H$ (the cut facet) for $H$ as above.

- If $\operatorname{dim} F>0$, choose a transversal affine subspace $N \subset V$ to $F$, i.e., complementary to the affine span aff $(F)$, and intersecting the relative interior $\stackrel{\circ}{F}$. Put $\widetilde{P}:=P \cap N$ and $\widetilde{F}:=F \cap N$. Then $L_{P}(F):=L_{\widetilde{P}}(\widetilde{F})\left(\right.$ so $\left.L_{P}(F)=N \cap P \cap H\right)$.

We note that the combinatorial type of a germ and that of a link is independent of all choices made in the construction. - In the literature on convex polytopes, a link of a vertex is usually called "vertex figure", and a link $L_{P}(F)$ of a face is called "face figure" or "quotient polytope", often noted $P / F$.

We recall the notion of the join of two "relatively skew" polytopes.

Definition 3.3. Let $Q_{1}, Q_{2}$ be disjoint polytopes in $V$ such that $\operatorname{dim} \operatorname{aff}\left(Q_{1} \cup Q_{2}\right)=$ $\operatorname{dim} Q_{1}+\operatorname{dim} Q_{2}+1$. Then their join $Q_{1} * Q_{2}$ is the convex hull of $Q_{1} \cup Q_{2}$ in $V$.

We note that $Q * \emptyset=Q$, and $Q_{1} * Q_{2}=Q_{2} * Q_{1}$. We remark that the join $Q_{1} * Q_{2}$ is the disjoint union of $Q_{1}, Q_{2}$, and all open segments $(x, y)$ joining points $x \in Q_{1}$ and $y \in Q_{2}$. We further remark that all faces of the join are of the form $F_{1} * F_{2}$, where $F_{i} \preceq Q_{i}$ is a (possibly empty) face, and that a link $L_{Q_{1} * Q_{2}}\left(Q_{1}\right)$ is combinatorially equivalent to $Q_{2}$. We denote with $\Pi(P):=P *\{\mathbf{a}\}$ for $\mathbf{a} \notin$ aff $(P)$ the pyramid with apex a and base $P$. An iterated pyramid $\Pi^{i}(P)$ for $i>0$ is thus a join $P * S_{i-1}$ with an $(i-1)$-simplex $S_{i-1}$, whereas $\Pi^{0}(P)=P$.

We now study the local geometry near a face $F$ of a polytope $P$ in $V$. For a given vertex $\mathbf{a} \in F$, we fix a nearby cutting hyperplane $H \subset V$. The cut facet $H \cap P$ is a link $L_{P}(\mathbf{a})$ of a in $P$, and its face $F \cap H$ is a link $L_{F}(\mathbf{a})$ of a relative to $F$.

Definition 3.4. A proper face $F \supsetneqq P$ of the polytope $P$ is called

- normally trivial (in $P$ ) at the vertex a if the link $L_{P}(\mathbf{a})$ is the join $L_{F}(\mathbf{a}) * S_{\mathbf{a}}$ with a suitable "complementary" face $S_{\mathbf{a}} \preceq L_{P}(\mathbf{a})$, and

- normally trivial (in $P$ ) if it is normally trivial at each of its vertices.

We remark that every vertex of a polytope $P$ is normally trivial as a face. If $\mathbf{a}$ is a simple vertex of $P$, then every face $F$ containing a is normally trivial at a: A link $L=L_{P}(\mathbf{a})$ of $\mathbf{a}$ in $P$ is a simplex, so for the face $F^{\prime}=L_{F}(\mathbf{a})$ of $L$, there is a unique complementary face; the latter being again a simplex, any link of $F$ in $P$ is a simplex. If $F \supsetneqq P$ is an edge or a facet of a three-dimensional polytope, then the converse holds: Normal triviality at a vertex $\mathbf{a}$ is equivalent to a being simple.

More generally, for a face $F \supsetneqq P$ that is normally trivial at the vertex $\mathbf{a}$, there is a unique face $F_{\mathbf{a}}^{\prime} \supsetneqq P$ "cutting out" the complementary face $S_{\mathbf{a}}$ in the link $L$, i.e., satisfying 
$S_{\mathbf{a}}=L_{P}(\mathbf{a}) \cap F_{\mathbf{a}}^{\prime}$. That face is complementary to $F$ at $\mathbf{a}$, i.e., we have $F \cap F_{\mathbf{a}}^{\prime}=\mathbf{a}$, $\operatorname{dim} F+\operatorname{dim} F_{\mathbf{a}}^{\prime}=\operatorname{dim} P$, and every edge emanating from a either lies in $F$ or in $F_{\mathbf{a}}^{\prime}$. Shifting the affine span of $F_{\mathbf{a}}^{\prime}$ to the relative interior of $F$ yields a transversal subspace $N$ to $F$ as in Def. 3.2. 2. As a consequence, the polytope $S_{\mathbf{a}}$ has the same combinatorial type as $L_{P}(F)$, so that type does not depend on the vertex $\mathbf{a} \in F$.

Normal triviality of a face yields a combinatorial local product structure:

Remark 3.5. Let $F \supsetneqq P$ be a normally trivial face with link $L=L_{P}(F)=N \cap P \cap H$ as in Def. 3.2. 2. Denote with $G=G_{P}(F)$ a corresponding germ and with $\pi: V \rightarrow N$ the (affine) projection onto $N$ along $\operatorname{aff}(F)$, i.e., collapsing $\operatorname{aff}(F)$ to a single point $\mathbf{v}_{0}$. Then $\pi$ induces a surjective map

$$
\left.\pi\right|_{G}: G \longrightarrow \Pi(L)
$$

onto the pyramid $\Pi(L):=G \cap N$ over $L$ with apex $\mathbf{v}_{0}$, mapping vertices onto vertices. Moreover, we obtain a bijection between the vertices of $G$ and the vertices of $F \times \Pi(L)$ as follows: A vertex $\mathbf{u}$ lying on the "ridge" $F$ of the "hip roof" $G$ is mapped to $\left(\mathbf{u}, \mathbf{v}_{0}\right)$, and a vertex $\mathbf{v}$ lying on the "bottom facet" $G \cap H$ (i.e., on the cut facet), being the end point of an edge emanating from a unique vertex $\mathbf{u} \in F$, is mapped to $(\mathbf{u}, \pi(\mathbf{v}))$. That map yields a combinatorial equivalence between the polytopes $G$ and $F \times \Pi(L)$.

If the link of a face is not a pyramid, then no vertex lying on that face is a simple point of the ambient polytope. This observation motivates the interest of the following concept that is essential for the inner loop, cf. $\mathrm{BreLu}_{2}$ 2.7:

Definition 3.6. $\quad$ 1. A (non-empty) polytope $P$ is called stout if it is not the pyramid over one of its facets.

2. A face $F \preceq P$ is called normally stout in $\boldsymbol{P}$ if one (and thus any) link $L=L_{P}(F)$ is stout.

Equivalently, a polytope $P$ is stout if for each facet $F$, there are at least two vertices of $P$ not lying on $F$. Hence, "stoutness" only depends on the combinatorial type and $\operatorname{dim} P \geq 2$ for a stout polytope $P$. In particular, a normally stout face $F \prec P$ always has codimension at least 3 .

The relation general versus stout polytopes is as follows:

Lemma 3.7. If a polytope $P$ is not a simplex, then it has exactly one maximal stout face $B \preceq P$. In particular $P$ is the iterated pyramid

$$
P=\Pi^{c}(B)=B * S_{c-1}
$$

(with $c:=\operatorname{codim}_{P} B \geqq 0$ ) over that "base face". Moreover, if $P$ is not stout (i.e., $c>0$ ), then the complementary simplex $S_{c-1}$ is the unique minimal normally stout face of $P$. 
Proof. If the polytope is stout, then there is nothing to show. The general case is seen by induction on $n:=\operatorname{dim} P \geqq 2$, with the case $n=2$ already being settled. For $n \geqq 3$, we may thus assume that $P$ is a pyramid $\Pi(F)=F *\{\mathbf{a}\}$ over one of its facets $F \prec P$. By induction hypothesis, the statement holds for that facet $F$. Since all faces of $P$ containing the apex a are pyramids, every stout face already lies in $F$. Hence, the unique maximal stout face $B$ of $F$ also is the unique maximal stout face of $P$.

The fundamental role played by normally stout faces in the present approach to the HRR is that they "witness" non-simplicity, cf. BreLu 2.9 :

Lemma 3.8. A polytope is simple if and only if it has no normally stout faces.

Proof. If a polytope is simple, then the links of all its faces are simplices, so no face is normally stout. On the other hand, a non-simple $n$-polytope $P$ has a vertex a $\in P$ that is incident to at least $n+1$ edges. A link $L=L_{P}(\mathbf{a})$ of that vertex is thus an $(n-1)$ polytope with more than $n$ vertices, so it is not a simplex. Hence, as seen above, it can be (uniquely) written as an iterated pyramid $L=\Pi^{c}(B)$ (for some $c \geqq 0$ ) over a stout base face $B \preceq L$. If $c=0$, i.e., $L=B$, then the vertex a already is a normally stout face of $P$. Otherwise we have $L=B * S_{c-1}$ with a (non-empty) simplex $S_{c-1} \supsetneqq L$ that is normally stout in $L$. Then the unique face $F \prec P$ cutting out the face $S_{c-1} \prec L$, i.e. such that $S_{c-1}=F \cap L$, is normally stout in $P$.

We may thus measure how "far" a polytope is from being simple:

Definition 3.9. The defect $\mu(P) \in \mathbf{N}$ of a polytope $P$ is defined as the number of normally stout faces of $P$.

We can restate Lemma 3.8 in these terms: A polytope $P$ is simple if and only if its defect vanishes, i.e., $\mu(P)=0$. - Pursuing the idea sketched at the beginning of this section, we now show that cutting off a minimal normally stout face brings us closer to "simplicity":

Lemma 3.10. Let $F \supsetneqq P$ be a normally stout face of minimal dimension, and let $R$ denote the residual polytope obtained by cutting off the face F from $P$. Then the "defect" satisfies

$$
\mu(R)=\mu(P)-1
$$

Proof. No proper face $F_{0} \supsetneqq G \cap R$ is normally stout in $R$, since $G \cap R \prec R$, as a cut facet, is normally trivial in $R$ and thus $L_{R}\left(F_{0}\right)=\Pi\left(L_{G \cap R}\left(F_{0}\right)\right)$. On the other hand there is a bijection between the faces of $P$ not contained in $F$ and the faces of $R$ not contained in $G \cap R$. Since corresponding faces have the same links and no proper face of $F$ is normally stout in $P$ by the minimality of $F$, we obtain $\mu(R)=\mu(P)-1$. 
Corollary 3.11. By finitely many successive cut-offs, every polytope is transformed into a simple one.

Proof. This follows from the above result together with the fact that a polytope $P$ with $\mu(P)=0$ is simple, cf. Lemma 3.8 .

So, finally, we are left with the problem to show that the HRR for the residual polytope $R$ obtained by cutting off a minimal normally stout face imply the HRR for the polytope $P$ itself. To that end, we have to study the "cut-off" part, namely, a germ of that face. With Remark 3.5 at our disposal, the following result turns out to be of crucial importance, cf. also $\mathrm{BreLu}_{2}$ 2.12:

Lemma 3.12. A normally stout face $F \prec P$ of minimal dimension is normally trivial and is itself a simple polytope.

Proof. We let $d:=\operatorname{dim} F$, the minimal dimension of any normally stout face. The case $d=0$ being trivial, we may assume $d>0$. Since an arbitrary vertex $\mathbf{a} \in F$ is neither simple nor normally stout in $P$, its link may be written in the form $L_{P}(\mathbf{a})=B * S_{c-1}$, where $B$ is stout and $c \geq 1$. The normally stout faces $F^{\prime} \supsetneqq P$ containing a correspond bijectively to the normally stout faces of $L_{P}(\mathbf{a})$ via $F^{\prime} \mapsto F^{\prime} \cap L_{P}(\mathbf{a})$. Since $F^{\prime}=F$ has minimal dimension, and $S_{c-1}$ is the unique normally stout face of $L_{P}(\mathbf{a})$ having minimal dimension, it follows that $L_{F}(\mathbf{a})=F \cap L_{P}(\mathbf{a})=S_{c-1}$, i.e., the point $\mathbf{a}$ is a simple vertex of $F$, and with $S_{\mathbf{a}}:=B \supsetneqq L_{P}(\mathbf{a})$ in Def. 3.4, the face $F$ is seen to be normally trivial in $P$ at $\mathbf{a}$.

\section{Intersection Cohomology of Fans}

In this section, we briefly recall the construction of the intersection cohomology of a (quasi-convex) fan $\Delta$, referring to $\mathrm{BBFK}_{2}$ or $\mathrm{BreLu}_{1}$ for details. All complete fans considered in the sequel occur as outer normal fans $\Delta(P)$ for a polytope $P \subset V$. Hence, we systematically consider fans in the dual $V^{*}$ of a given vector space $V$. We are not going to deal with non-polytopal complete fans.

4.A The fan space: Motivated by the coarse "toric topology" on a toric variety given by torus-invariant open sets, we consider a fan $\Delta$ in $V^{*}$ as a finite topological space with the subfans as open subsets. The "affine" fans

$$
\langle\sigma\rangle:=\{\sigma\} \cup \partial \sigma \preceq \Delta \quad \text { with boundary fan } \quad \partial \sigma:=\{\tau \in \Delta ; \tau \supsetneqq \sigma\}
$$

form a basis of the fan topology by open sets that cannot be covered by smaller ones. Here $\preceq$ means that a cone is a face of another cone or that a set of cones is a subfan of 
some other fan. In fact, by abuse of notation, we often write $\sigma$ instead of $\langle\sigma\rangle$, if there is no danger of confusion.

4.B Sheaves: Sheaf theory on a fan (space) $\Delta$ is particularly simple since a presheaf given on the basis uniquely extends to a sheaf. In order to simplify notation, given a sheaf $\mathcal{F}$ on $\Delta$, we write

$$
F_{\Lambda}:=\mathcal{F}(\Lambda)
$$

for the set of sections on the open subset (i.e., subfan) $\Lambda \preceq \Delta$. Then a sheaf $\mathcal{F}$ is flabby if and only if each restriction homomorphism

$$
\varrho_{\partial \sigma}^{\sigma}: F_{\sigma} \rightarrow F_{\partial \sigma}
$$

is surjective.

Here are the two most important examples:

1. The structure sheaf $\mathcal{A}$ of $\Delta$ is defined by

$$
A_{\sigma}:=S\left(\operatorname{span}(\sigma)^{*}\right)
$$

the graded algebra of real-valued polynomial functions on the subspace $\operatorname{span}(\sigma) \subset$ $V^{*}$ or rather on $\sigma$ itself, the homomorphisms $\varrho_{\tau}^{\sigma}: A_{\sigma} \longrightarrow A_{\tau}$ for $\tau \preceq \sigma$ being the restriction of functions. Hence, for $\Lambda \preceq \Delta$, the global sections $\in A_{\Lambda}$ are the $\Lambda$-conewise polynomial functions $|\Lambda| \longrightarrow \mathbf{R}$. The grading is chosen to be twice the standard grading, e.g. cone-wise linear functions get the degree 2.

The structure sheaf $\mathcal{A}$ is flabby if and only if $\Delta$ is a simplicial fan.

2. The "equivariant" intersection cohomology sheaf $\mathcal{E}$ (also called "minimal extension sheaf" in $\mathrm{BBFK}_{2}$ ) is the "smallest" flabby sheaf of graded $\mathcal{A}$-modules on $\Delta$ such that $E_{\sigma}$ is a finitely generated free $A_{\sigma}$-module for every cone $\sigma \in \Delta$, and $E_{o}=A_{o}=\mathbf{R}$ for the zero cone $o:=\{0\}$.

Let us explain the minimality condition in "smallest": Let

$$
A:=S(V)=S\left(\left(V^{*}\right)^{*}\right)
$$

denote the (even-graded) algebra of polynomial functions on the vector space $V^{*}$ (so in particular, $A_{\sigma}=A$ for an $n$-cone $\sigma$, and for any fan $\Lambda$ both, $A_{\Lambda}$ and $E_{\Lambda}$ are graded $A$-modules in a natural way). Furthermore, let

$$
\mathfrak{m}:=A^{>0}
$$


denote the unique homogeneous maximal ideal of the graded algebra $A$. Then, given a graded $A$-module, we define its reduction $\bar{M}$, a graded real vector space, by

$$
\bar{M}:=(A / \mathfrak{m}) \otimes_{A} M .
$$

Since $\mathcal{E}$ is flabby, the reduced restriction

$$
\bar{\varrho}_{\partial \sigma}^{\sigma}: \bar{E}_{\sigma} \rightarrow \bar{E}_{\partial \sigma}
$$

is also surjective. Requiring it to be even an isomorphism means minimizing the rank of the free $A_{\sigma}$-module $E_{\sigma}$. Note that, on the other hand, the surjectivity of $\bar{\varrho}_{\partial \sigma}^{\sigma}$ already implies that of $\varrho_{\partial \sigma}^{\sigma}$.

The above conditions determine $\mathcal{E}$ up to isomorphy of graded $\mathcal{A}$-modules, and in particular we see that $\mathcal{E} \cong \mathcal{A}$ iff $\Delta$ is simplicial.

4.C The intersection cohomology $I H(\Delta)$ of a complete (or more general: "quasiconvex") fan is defined as the graded vector space

$$
I H(\Delta):=\bar{E}_{\Delta}
$$

4.D Quasi-convex fans: We call a fan quasi-convex if it is purely $n$-dimensional, i.e., all maximal cones are $n$-dimensional, and the support $|\partial \Delta|$ of its boundary subfan is a real homology manifold or empty. Here $\partial \Delta \preceq \Delta$ is the subfan generated by those $(n-1)$ cones which are a facet of exactly one $n$-cone in $\Delta$. In fact, quasi-convex fans $\Delta$ are characterized by the fact that $E_{\Delta}$ is a (finitely generated) free $A$-module, cf. $\mathrm{BBFK}_{2}$, 4.1 and 4.4 .

So in particular fans with convex or coconvex support (i.e., $V^{*} \backslash|\Delta|$ is convex) as well as stars of cones in a complete fan provide examples of such fans. Furthermore if $\Lambda \preceq \Delta$ is a quasi-convex subfan of the complete fan $\Delta$, we denote with $\Lambda^{c} \preceq \Delta$ its (quasi-convex) complementary subfan, i.e., $\Lambda^{c}$ is the subfan generated by the $n$-cones in $\Delta \backslash \Lambda$.

4.E Outer normal fan and Lefschetz Operator: Any $n$-polytope $P \subset V$ induces a fan $\Delta=\Delta(P)$ in $V^{*}$ together with a strictly convex $\Delta$-conewise linear function $\psi: V^{*} \longrightarrow \mathbf{R}$ as follows: For any facet $F \preceq_{1} P$ choose an "outer normal vector" $\mathbf{n}_{F} \in V^{*} \backslash\{0\}$, i.e., $\left.\mathbf{n}_{F}\right|_{F} \equiv$ const $\geq\left.\mathbf{n}_{F}\right|_{P}$, and denote with $\nu(F):=\mathbf{R}_{\geq 0} \mathbf{n}_{F}$ the associated "outer normal ray" of the facet $F$. To any face $G \preceq P$, we associate a cone $\sigma(G) \subset V^{*}$ as follows:

$$
\sigma(G):=\sum_{G \preceq F \preceq{ }_{1} P} \nu(F) .
$$

Note in particular that $\sigma(P)=o:=\{0\} \subset V^{*}$, the zero cone. Then the outer normal fan $\Delta(P)$ is defined as

$$
\Delta(P):=\{\sigma(G) ; G \preceq P\}
$$


We remark that $\Delta(P)$ is simplicial, iff $P$ is simple.

Denote $\mathbf{v}_{1}, \ldots, \mathbf{v}_{r} \in V$ the vertices of $P$. Then $\sigma_{i}:=\sigma\left(\left\{\mathbf{v}_{i}\right\}\right), i=1, \ldots, r$ are the $n$ dimensional cones in $\Delta$. Denote $\psi_{i} \in\left(V^{*}\right)^{*}$ the image of $\mathbf{v}_{i}$ with respect to the biduality isomorphism $V \longrightarrow\left(V^{*}\right)^{*}$. Then

$$
\left.\psi\right|_{\sigma_{i}}:=\psi_{i}
$$

defines a strictly convex conewise linear function $\psi \in A_{\Delta(P)}^{2}$. Put $\Delta:=\Delta(P)$. The multiplication map

$$
\mu_{\psi}: E_{\Delta} \longrightarrow E_{\Delta}, f \longmapsto \psi f
$$

induces a degree 2 map

$$
L:=\bar{\mu}_{\psi}: \bar{E}_{\Delta}=I H(\Delta(P)) \longrightarrow \bar{E}_{\Delta}=I H(\Delta(P)),
$$

the "Lefschetz operator". We remark that $\Delta(P+\mathbf{a})=\Delta(P)$ for $\mathbf{a} \in V$ with the same Lefschetz operator, since the correponding strictly convex functions only differ by the "globally linear" function $\mathbf{a} \in V \cong\left(V^{*}\right)^{*}=A^{2}$.

If $\operatorname{aff}(P) \neq V$, the above constructions apply mutatis mutandis in order to give a fan $\Delta(P)$ in $V^{*} / \operatorname{aff}_{0}(P)^{\perp}$, with the subspace $\operatorname{aff}_{0}(P):=\operatorname{aff}(P)-\mathbf{a}, \mathbf{a} \in \operatorname{aff}(P)$, as well as a Lefschetz operator on $I H(\Delta(P))$.

4.F The intersection product: For details cf. $\left.\mathrm{BBFK}_{3}\right]$. We need this notation for a sheaf $\mathcal{F}$ on a quasi-convex fan $\Delta$ : The module $F_{(\Delta, \partial \Delta)} \subset F_{\Delta}$ of "sections with compact support on $\Delta "$ is defined as

$$
F_{(\Delta, \partial \Delta)}:=\operatorname{ker}\left(\varrho_{\partial \Delta}^{\Delta}\right)=\left\{f \in F_{\Delta} ;\left.f\right|_{\partial \Delta}=0\right\},
$$

such that for $\Delta \preceq \Lambda$, there is a natural inclusion $F_{(\Delta, \partial \Delta)} \subset F_{\Lambda}$ by trivial extension of sections. In order to discuss the intersection product, we have to fix a volume form $\omega \in \operatorname{det} V:=\bigwedge^{n} V$ on $V^{*}$. If the fan $\Delta$ is simplicial, we can, following [Bri], define a graded $A$-linear "evaluation map"

$$
\varepsilon: A_{(\Delta, \partial \Delta)} \longrightarrow A[-2 n]
$$

as follows: For each $n$-cone $\sigma$, we denote $g_{\sigma} \in A_{(\sigma, \partial \sigma)}^{2 n} \subset A_{\sigma}=A$ the unique non-trivial function $\geq 0$, which is the product of linear forms in $A^{2} \cong V$, whose wedge product agrees, up to sign, with $\omega$. Then the map $\varepsilon$ is the composite

$$
E_{(\Delta, \partial \Delta)} \cong A_{(\Delta, \partial \Delta)} \subset \bigoplus_{\sigma \in \Delta^{n}} A_{\sigma} \longrightarrow Q(A), f=\left(f_{\sigma}\right)_{\sigma \in \Delta^{n}} \longmapsto \sum_{\sigma \in \Delta^{n}} \frac{f_{\sigma}}{g_{\sigma}},
$$

mapping $A_{(\Delta, \partial \Delta)}$ onto $A \subset Q(A)$. We remark that any (graded) $A$-linear map $A_{(\Delta, \partial \Delta)} \longrightarrow$ $A[-2 n]$ is a scalar multiple of $\varepsilon$, and that a multiplication of $\omega$ with a scalar $\lambda \in \mathbf{R}$ results in a multiplication of $\varepsilon$ with $|\lambda|$. 
The intersection product then is the composite

$$
. . \cap \ldots: A_{\Delta} \times A_{(\Delta, \partial \Delta)} \stackrel{\text { mult }}{\longrightarrow} A_{(\Delta, \partial \Delta)} \stackrel{\varepsilon}{\longrightarrow} A[-2 n]
$$

of the multiplication of functions and the evaluation map $\varepsilon: A_{(\Delta, \partial \Delta)} \longrightarrow A[-2 n]$. In the general case the definition uses the dual sheaf $\mathcal{D E}$ of $\mathcal{E}$, cf. $\left[\mathrm{BBFK}_{3}\right.$. Its sections over a cone $\sigma \in \Delta$ are

$$
(\mathcal{D E})_{\sigma}:=\operatorname{Hom}\left(E_{(\sigma, \partial \sigma)}, A_{\sigma}\right) \otimes \operatorname{det} V_{\sigma},
$$

with $V_{\sigma}:=V / \operatorname{span}(\sigma)^{\perp} \cong \operatorname{span}(\sigma)^{*}$. The determinant factor produces a degree shift ( $V_{\sigma}=A_{\sigma}^{2}$ being of weight 2) and plays an important role in the definition of the restriction homomorphisms $(\mathcal{D E})_{\sigma} \longrightarrow(\mathcal{D E})_{\tau}$ for $\tau \preceq \sigma$. Here it is necessary to fix an orientation of $\operatorname{span}(\sigma)$ for every cone $\sigma \in \Delta$, with the $n$-cones getting the orientation defined by the volume form $\omega \in \operatorname{det} V$. Then the defining formula holds even globally:

$$
(\mathcal{D E})_{\Delta} \cong \operatorname{Hom}\left(E_{(\Delta, \partial \Delta)}, A\right) \otimes \operatorname{det} V \cong \operatorname{Hom}\left(E_{(\Delta, \partial \Delta)}, A[-2 n]\right),
$$

where the second isomorphy uses the isomorphism $\operatorname{det} V \cong \mathbf{R}, \omega \mapsto 1$. Furthermore there are natural isomorphisms $\mathcal{E} \cong \mathcal{D E}$ - in fact, the naturality is obtained only with the HLT for fans in lower dimensions - and $E_{\Delta} \cong(\mathcal{D E})_{\Delta}$, whence we finally obtain the intersection product

$$
. . \cap \ldots: E_{\Delta} \times E_{(\Delta, \partial \Delta)} \longrightarrow A[-2 n],
$$

which uniquely extends to a map

$$
. . \cap \ldots: E_{\Delta} \times E_{\Delta} \longrightarrow A f^{-1}[-2 n],
$$

where $f \in A$ is a minimal square free product of linear forms in $A^{2}=V$ with $\left.f\right|_{\partial \Delta}=0$. If we apply that to the subfans $\langle\sigma\rangle$ with an $n$-cone $\sigma \in \Delta$ we obtain a formula representing the intersection product of two sections $f \in E_{\Delta}$ and $g \in E_{(\Delta, \partial \Delta)}$ as a sum of local contributions:

$$
f \cap g=\sum_{\sigma \in \Delta^{n}} f_{\sigma} \cap g_{\sigma} \in A
$$

with $f_{\sigma}:=\left.f\right|_{\sigma}, g_{\sigma}:=\left.g\right|_{\sigma}$, but note that in general $f_{\sigma} \cap g_{\sigma} \in Q(A)$ does not belong to $A$.

There is another way to obtain the intersection product (cf. $\left.\mathrm{BBFK}_{3}, 4\right)$ : Take a simplicial refinement $\iota: \Sigma \longrightarrow \Delta$ and realize $\mathcal{E}$ as a direct summand of $\iota_{*}(\mathcal{A})$, where $\mathcal{A}$ denotes the structure sheaf of the fan $\Sigma$, (cf. $\left.\mathrm{BBFK}_{2}, 2.5\right)$ - the corresponding inclusion then is also called a direct embedding. Then the composition

$$
E_{\Delta} \times E_{(\Delta, \partial \Delta)} \hookrightarrow A_{\Sigma} \times A_{(\Sigma, \partial \Sigma)} \stackrel{\text { mult }}{\longrightarrow} A_{(\Sigma, \partial \Sigma)} \stackrel{\varepsilon}{\longrightarrow} A[-2 n]
$$

of the induced embeddings and the intersection product on $\Sigma$ provides the intersection product on $\Delta$. 
A third possibility is to mimic the multiplication of functions (cf. $\left.\mathrm{BBFK}_{3}, 4\right)$ : Choose an "internal intersection product", i.e., any symmetric $\mathcal{A}$-bilinear sheaf homomorphism $\beta: \mathcal{E} \times \mathcal{E} \longrightarrow \mathcal{E}$ extending the multiplication of functions on the 2-skeleton - but note that its construction involves choices and is not natural. On the other hand there is a distinguished section $1 \in E_{\Delta}$ and its image with respect to the isomorphism

$$
E_{\Delta} \stackrel{\cong}{\longrightarrow}(\mathcal{D E})_{\Delta} \cong \operatorname{Hom}_{A}\left(E_{(\Delta, \partial \Delta)}, A[-2 n]\right)
$$

provides an evaluation map $\varepsilon: E_{(\Delta, \partial \Delta)} \longrightarrow A[-2 n]$. Then if we take the composite

$$
E_{\Delta} \times E_{(\Delta, \partial \Delta)} \stackrel{\beta}{\longrightarrow} E_{(\Delta, \partial \Delta)} \stackrel{\varepsilon}{\longrightarrow} A[-2 n],
$$

we finally once again obtain the intersection product!

\section{$5 \quad$ HRR for special $n$-polytopes}

\subsection{HRR for pyramids}

Proposition 5.1. If the $H R R$ hold for polytopes in dimension $<n$, then also for any $n$-dimensional pyramid $P=\Pi(Q)$ over some $(n-1)$-polytope $Q$.

Proof. We may assume that $0 \in V$ is the apex of our pyramid, i.e. $\Pi(Q)=Q *\{0\}$. Let $\Delta:=\Delta(\Pi(Q))$ and denote $\sigma:=\sigma(\{0\}) \in \Delta$ the cone corresponding to the apex 0 of the pyramid. Then the complementary fan $\Delta_{0}:=\Delta \backslash\{\sigma\}=\langle\sigma\rangle^{c}$ satisfies

$$
\Delta_{0}=\operatorname{st}(\nu(Q))=\partial \sigma+\nu(Q):=\partial \sigma+\langle\nu(Q)\rangle
$$

with the outer normal ray $\nu(Q)$ of $Q \preceq_{1} P=\Pi(Q)$, and $\left.\psi\right|_{\sigma}=0$ resp. $\psi \in A_{\left(\Delta_{0}, \partial \Delta_{0}\right)}^{2} \subset$ $A_{\Delta}^{2}$. We regard the exact sequence

$$
0 \longrightarrow E_{(\sigma, \partial \sigma)} \longrightarrow E_{\Delta} \longrightarrow E_{\Delta_{0}} \longrightarrow 0
$$

It even splits, since $E_{\Delta_{0}}$ is free, the fan $\Delta_{0}$ being quasi-convex. Thus, there is a corresponding exact sequence

$$
0 \longrightarrow I H(\sigma, \partial \sigma) \longrightarrow I H(\Delta) \longrightarrow I H\left(\Delta_{0}\right) \longrightarrow 0
$$

with $I H^{q}(\sigma, \partial \sigma)=0$ for $q \leq n$, since HLT holds for fans in dimension $<n$, cf. $\mathrm{BBFK}_{2}, 1.8$; so for $k \geq 0$ the restriction from $\Delta$ to $\Delta_{0}$ induces an isomorphism

$$
I H^{n-k}(\Delta) \stackrel{\cong}{\longrightarrow} I H^{n-k}\left(\Delta_{0}\right) \cong I H^{(n-1)-(k-1)}(\Delta(Q))
$$


Let us comment here on the second isomorphy: The outer normal fan $\Delta(Q)$ is a fan in $W:=V^{*} / \mathbf{R n}_{Q}$, and the quotient projection $\pi: V^{*} \longrightarrow W$ induces a fan map $\Delta_{0} \longrightarrow$ $\Delta(Q)$. Then, with $B:=S\left(W^{*}\right) \subset A=S\left(\left(V^{*}\right)^{*}\right)$ we have

$$
E_{\Delta_{0}} \cong A \otimes_{B} E_{\Delta(Q)}
$$

whence the last isomorphism. The dual picture looks as follows

$$
I H^{n+k}(\Delta) \cong I H^{n+k}\left(\Delta_{0}, \partial \Delta_{0}\right) \cong I H^{n+k-2}\left(\Delta_{0}\right) \cong I H^{(n-1)+(k-1)}(\Delta(Q))
$$

Here the second isomorphism is the "Thom isomorphism", the isomorphism induced by:

$$
E_{\Delta_{0}} \stackrel{\cong}{\longrightarrow} E_{\left(\Delta_{0} . \partial \Delta_{0}\right)}, f \mapsto \psi f .
$$

Analogously with $k+2$ instead of $k$ it is like this:

$$
I H^{n+k+2}(\Delta) \cong I H^{(n-1)+(k-1)+2}(\Delta(Q)) .
$$

For $k>0$, these isomorphisms transform

$$
L^{k}: I H^{n-k}(\Delta) \longrightarrow I H^{n+k}(\Delta)
$$

into

$$
L^{k-1}: I H^{(n-1)-(k-1)}(\Delta(Q)) \longrightarrow I H^{(n-1)+(k-1)}(\Delta(Q)) .
$$

This gives the HLT for $\Delta$. Now let us look at the HRR: The homomorphism

$$
L^{k+1}: I H^{n-k}(\Delta) \longrightarrow I H^{n+k+2}(\Delta)
$$

corresponds to

$$
L^{(k-1)+1}: I H^{(n-1)-(k-1)}(\Delta(Q)) \longrightarrow I H^{(n-1)+(k-1)+2}(\Delta(Q)) .
$$

So

$$
I P^{n-k}(\Delta) \cong I P^{(n-1)-(k-1)}(\Delta(Q)) \quad \text { for } k>0,
$$

while for $k=0$ there is no contribution: $I P^{n}(\Delta)=0$ because of $L^{0}=$ id. Now the above isomorphism respects the Hodge-Riemann forms, if we endow $V^{*} / \mathbf{R n}_{Q}$ with the volume form $\eta$, such that $q^{*}(\eta) \wedge \psi_{\tau}=\omega$ with the volume form $\omega$ of $V^{*}$, the quotient map $q: V^{*} \longrightarrow V^{*} / \mathbf{R n}_{Q}$ and $\psi_{\tau}=\left.\psi\right|_{\tau} \in A^{2}=\left(V^{*}\right)^{*}$ with an $n$-cone $\tau \in \Delta_{0}$. So the HRR hold for $\Pi(Q)$, since they do for $Q$. 


\subsection{The Künneth formula}

We want to show that the product $S \times P_{0}$ of a "HRR polytope" $P_{0}$ with a simple factor $S$ again has the "HRR property". We start with discussing the intersection cohomology, endowed with the intersection product.

Proposition 5.2. Let $P=S \times P_{0}$ be a polytope in $V \times W$ with a simple factor $S$, and let $\Delta=\Sigma \oplus \Delta_{0}$ be the corresponding decomposition of the respective outer normal fans. Then there is a natural isomorphism

$$
I H(\Delta) \stackrel{\cong}{\longrightarrow} I H(\Sigma) \otimes_{\mathbf{R}} I H\left(\Delta_{0}\right)
$$

of graded vector spaces endowed with the intersection forms.

Proof. We let $A=S(V)$ and $B=S(W)$ denote the algebra of polynomials on $V^{*}$ and on $W^{*}$, respectively. Disregarding the intersection products, the isomorphism is seen as follows: Since $S$ is simple, the fan $\Sigma$ is simplicial. Hence, assigning to a cone $\delta=\sigma \times \delta_{0}$ in $\Delta=\Sigma \oplus \Delta_{0}$ the $A_{\delta}$-module

$$
E_{\delta}:=A_{\sigma} \otimes_{\mathbf{R}} E_{\delta_{0}}
$$

defines a minimal extension sheaf on $\Delta$, as follows from an iterated application of Lemma 1.5 in $\mathrm{BBFK}_{2}$. Since the functor $A_{\sigma} \otimes \ldots$ is exact we obtain

$$
E_{\sigma \times \Delta_{0}} \cong A_{\sigma} \otimes E_{\Delta_{0}}
$$

for each $\sigma \in \Sigma$. Using the analogous argument with the functor $\ldots \otimes E_{\Delta_{0}}$, we obtain

$$
E_{\Delta} \cong A_{\Sigma} \otimes E_{\Delta_{0}}
$$

Since both, $A_{\Sigma}$ and $E_{\Delta_{0}}$, are free modules over their base rings $A$ and $B$, respectively, the latter isomorphism descends to the level of intersection cohomology.

It remains to check the compatibility with the intersection products. We first assume that the fan $\Delta_{0}$ is simplicial, too. In that case, up to suitable shifts, the tensor product of the evaluation maps $A_{\Sigma} \rightarrow A$ and $A_{\Delta_{0}} \rightarrow B$ defines the evaluation map

$$
A_{\Delta} \cong A_{\Sigma} \otimes A_{\Delta_{0}} \longrightarrow A \otimes B
$$

associated to the product of the pertinent volume forms on $V$ and on $W$, respectively. This implies the compatibility.

If $\Delta_{0}$ is non-simplicial, we choose a simplicial subdivision $\iota: \widehat{\Delta}_{0} \rightarrow \Delta_{0}$ and a direct embedding $\mathcal{E} \hookrightarrow \iota_{*}(\widehat{\mathcal{A}})$ of the intersection cohomology sheaf $\mathcal{E}$ on $\Delta_{0}$ into the direct image of the structure sheaf $\widehat{\mathcal{A}}$ on $\widehat{\Delta}_{0}$. It induces a direct embedding on $\Delta=\Sigma \times \Delta_{0}$. Since these embeddings provide the respective intersection products on $E_{\Delta_{0}}$ and on $E_{\Delta}$, the compatibility holds. 
To show the HRR property, we need some purely algebraic considerations. In that framework, it is convenient to make degrees symmetric by a shift: Instead of $I H(\Delta)$, graded in even degrees ranging from 0 to $2 n$ and endowed with the intersection pairing and the Lefschetz operator, we consider the following

Abstract HR setup 5.3. Let

$$
W:=\bigoplus_{k=-m}^{m} W^{k}
$$

be a finite dimensional graded vector space endowed with the following structures:

- A non-degenerate symmetric bilinear form, also called the "intersection form",

$$
\left\langle{ }_{-},{ }_{-}\right\rangle: W \times W \longrightarrow \mathbf{C}
$$

of total degree 0 satisfying

$$
\left\langle W^{-k}, W^{k}\right\rangle \subset i^{k} \mathbf{R},
$$

- the structure of a graded module over the polynomial ring $\mathbf{R}[L]$ with $\operatorname{deg} L=2$ such that the "Lefschetz operator" $\mu_{L}:=L \cdot \ldots$ is self-adjoint with respect to the above form.

For the convenience of notation we simply write $L$ instead of $\mu_{L}$. These data give rise to "HR-forms" $s_{k}(x, y):=\left(x, L^{k} y\right)$ on $W^{-k}$, and furthermore, to "primitive subspaces"

$$
P\left(W^{-k}\right):=\operatorname{ker}\left(L^{k+1}: W^{-k} \rightarrow W^{k+2}\right) .
$$

Definition 5.4. A graded $\mathbf{R}[L]$-module $W$ endowed with such a structure is called an $\mathbf{H R -}$ module if the restriction of $i^{k} s_{k}$ to the primitive subspace $P\left(W^{-k}\right)$ is positive definite.

We note that obviously, an HR-module satisfies $\operatorname{dim} W^{k}=\operatorname{dim} W^{-k}$ ("numerical Poincaré duality").

The following HR-modules $A_{m}$ (for $m \in \mathbf{N}$ ) are the simple ones. They are defined by putting

$$
A_{m}^{k}:= \begin{cases}\mathbf{R} & \text { for }-m \leqq k \leqq m \text { with } k \equiv m \bmod 2, \\ 0 & \text { otherwise. }\end{cases}
$$

with the intersection form mapping the pair $(1,1) \in A_{m}^{-k} \times A_{m}^{k}$ to $\langle 1,1\rangle=(-i)^{m}$, and the "Lefschetz operator" $L: A_{m}^{k} \rightarrow A_{m}^{k+2}$ mapping $1 \mapsto 1$, whenever that makes sense.

Remark 5.5. i) Every HR-module is isomorphic to a direct sum of modules $A_{m}$.

ii) A graded $\mathbf{R}[L]$-submodule $U \subset W$ of an HR-module $W$ is again an HR-module iff it satisfies numerical Poincaré duality $\operatorname{dim} U^{-k}=\operatorname{dim} U^{k}$. 
The link with intersection cohomology of polytopes is provided as follows:

Remark 5.6. Let $P$ be an n-polytope and put $\Delta:=\Delta(P)$. Endow the graded $\mathbf{R}$-vector space $W(P):=I H(\Delta)[-n]$ (i.e., having weight spaces $W^{k}(P):=I H^{n+k}(\Delta)$ for $-n \leqq$ $k \leqq n)$ with the intersection form, multiplied by $(-i)^{n}$, and put $L$ to be the Lefschetz operator. Then the polytope $P$ satisfies HRR iff $W(P)$ is an HR-module. Furthermore $W\left(S_{n}\right) \cong A_{n}$ holds for the n-simplex $S_{n}$.

We now state and prove the "Künneth theorem" for HR-modules.

Proposition 5.7. Let $W, W^{\prime}$ be HR-modules. Then both, $W \oplus W^{\prime}$ and $W \otimes W^{\prime}$ are HR-modules, where the action of $L$ on $W \otimes W^{\prime}$ is given by $L(x \otimes y):=L x \otimes y+x \otimes L y$.

Proof. The first part of the statement being obvious, we only have to consider the tensor product. Since both, $W$ and $W^{\prime}$ are direct sums of modules of type $A_{n}$ and the tensor product commutes with direct sums, it suffices to look at $A_{n} \otimes A_{m}$. As a first step, a direct computation shows that, for $n \geq 1$,

$$
A_{n} \otimes A_{1} \cong A_{n+1} \oplus A_{n-1}
$$

is an HR-module. By induction on $n$, it follows that

$$
\left(A_{1}\right)^{\otimes n} \cong A_{n} \oplus R
$$

also is an HR-module, where the "remainder" $R$ is a direct sum of terms $A_{m}$ with $m<n$ of the same parity as $n$. Hence the graded vector space

$$
B:=A_{n} \otimes A_{m}
$$

is an $\mathbf{R}[L]$-submodule of the HR-module $C:=\left(A_{1}\right)^{\otimes(n+m)}$. Satisfying numerical Poincaré duality $\operatorname{dim} B^{-k}=\operatorname{dim} B^{k}$, it is an HR-module itself.

Corollary 5.8. If, in the situation of Proposition 5.2, the polytope $P_{0}$ satisfies $H R R$, then so does $P=S \times P_{0}$.

Proof. According to 5.2 and in the notation of [5.6, the graded $\mathbf{R}[L]$-module $W(P)$ can be written as

$$
W(P) \cong W(S) \otimes W\left(P_{0}\right) ;
$$

hence the claim follows from 5.7 and the HRR for the simple polytope $S$. 


\subsection{Transversal Cuttings}

Proposition 5.9. If the affine hyperplane $H$ cuts $P$ transversally into the polytopes $P_{1}$ and $P_{2}$, i.e., $H$ has nonempty intersection with the relative interior of $P$ and does not contain vertices of $P$, then the validity of $H R R$ for $P_{1}$ and $P_{2}$ and for lower dimensional polytopes implies HRR for $P$.

Proof. Let us write

$$
F_{i}:=P \cap H \preceq P_{i},
$$

using the index $i=1,2$ in order to indicate when $P \cap H$ should be considered as a facet of $P_{i}$.

(A) Fans involved. First of all let, as usual,

$$
\Delta:=\Delta(P), \text { and put } \Delta_{i}:=\Delta\left(P_{i}\right) \text { for } i=1,2 .
$$

Secondly, we consider the "intermediate" polytope $Q$ cut out from $P$ by $H$ and a nearby parallel hyperplane. Its outer normal fan $\Delta(Q)$ is obtained by just putting together the stars

$$
\Lambda_{i}:=\operatorname{st}\left(\nu\left(F_{i}\right)\right) \preceq \Delta_{i}
$$

of the outer normal ray to the "cut" facet with respect to the fans $\Delta_{i}$ to a new complete fan

$$
\Lambda:=\Lambda_{1} \cup \Lambda_{2}=\Delta(Q) .
$$

Finally, we let $\Phi:=\Delta\left(F_{1}\right)=\Delta\left(F_{2}\right)$ denote the outer normal fan of $F:=F_{1}=F_{2}$ in $W:=V^{*} /\left(\mathbf{R} \cdot \mathbf{n}_{F_{i}}\right)$ (note that $\left.\mathbf{n}_{F_{2}}=-\mathbf{n}_{F_{1}}\right)$.

(B) Gluing of $\boldsymbol{I} \boldsymbol{H}$. Let $\mathcal{G}$ and $\mathcal{E}$ denote the respective intersection cohomology sheaves on $\Phi$ and $\Lambda$. The projection $\pi: V^{*} \rightarrow W$ induces a map of fans $\Lambda \rightarrow \Phi$, and we have $\mathcal{E} \cong \pi^{*}(\mathcal{G})$. In particular, writing $B:=S\left(W^{*}\right) \subset A:=S\left(\left(V^{*}\right)^{*}\right)$, there is a natural injection

$$
A \otimes_{B} G_{\Phi} \hookrightarrow E_{\Lambda}
$$

which after restriction to the subfans $\Lambda_{i} \preceq \Lambda$ gives isomorphisms

$$
E_{\Lambda_{1}} \stackrel{\cong}{\longleftarrow} A \otimes_{B} G_{\Phi} \stackrel{\cong}{\longrightarrow} E_{\Lambda_{2}} .
$$

Denote with

$$
S: E_{\Lambda_{1}} \stackrel{\cong}{\longrightarrow} E_{\Lambda_{2}}
$$

the resulting $A$-module isomorphism. Now consider the exact sequence

$$
0 \longrightarrow K \longrightarrow E_{\Delta_{1}} \oplus E_{\Delta_{2}} \longrightarrow E_{\Lambda_{2}} \longrightarrow 0,
$$


where the second nontrivial map sends $\left(f_{1}, f_{2}\right)$ to $S\left(\left.f_{1}\right|_{\Lambda_{1}}\right)-\left.f_{2}\right|_{\Lambda_{2}}$, and $K \subset E_{\Delta_{1}} \oplus E_{\Delta_{2}}$ is the kernel of that map. Since $E_{\Lambda_{2}}$ is a free $A$-module and

$$
I H\left(\Lambda_{i}\right) \cong I H(\Phi)
$$

the exact sequence (2) induces the exact sequence

$$
0 \longrightarrow \bar{K} \longrightarrow I H\left(\Delta_{1}\right) \oplus I H\left(\Delta_{2}\right) \longrightarrow I H(\Phi) \longrightarrow 0
$$

Furthermore we need

$$
0 \longrightarrow D \longrightarrow K \longrightarrow E_{\Delta} \longrightarrow 0
$$

where the second nontrivial map is gluing of sections: The fan $\Delta$ is the union

$$
\Delta=\Lambda_{1}^{c} \cup \Lambda_{2}^{c}
$$

of the complementary subfans $\Lambda_{i}^{c} \preceq \Delta_{i}$ of $\Lambda_{i} \preceq \Delta_{i}$, i.e.,

$$
\Delta_{i}=\Lambda_{i} \cup \Lambda_{i}^{c}
$$

with the quasi-convex fans $\Lambda_{i}$ and $\Lambda_{i}^{c}$ intersecting only in their common boundary fan and, in particular, $\left|\Lambda_{i}\right|=\left|\Lambda_{j}^{c}\right|$ for $j \neq i$. Now for a pair $\left(f_{1} . f_{2}\right) \in K \subset E_{\Delta_{1}} \oplus E_{\Delta_{2}}$ we define its image as the section $f \in E_{\Delta}$ satisfying $\left.f\right|_{\Lambda_{i}^{c}}:=\left.f_{i}\right|_{\Lambda_{i}^{c}}$. The kernel $D$ then satisfies

$$
D=\left\{(h, S(h)) ; h \in E_{\left(\Lambda_{1}, \partial \Lambda_{1}\right)}\right\} \cong E_{\left(\Lambda_{1}, \partial \Lambda_{1}\right)} .
$$

(C) Gluing of the intersection product. The exact sequence (4) yields an isomorphism

$$
E_{\Delta} \cong K / D
$$

in fact that quotient representation holds even with respect to the intersection pairings on $E_{\Delta}$ and $K \subset E_{\Delta_{1}} \oplus E_{\Delta_{2}}$ : Consider two pairs $\left(f_{1}, f_{2}\right),\left(g_{1}, g_{2}\right) \in K$ and denote $f, g \in E_{\Delta}$ their respective images in $E_{\Delta}$. Then in $Q(A)$ we obtain

$$
\begin{gathered}
\left(f_{1}, f_{2}\right) \cap\left(g_{1}, g_{2}\right)=f_{1} \cap g_{1}+f_{2} \cap g_{2} \\
=f_{1} \cap_{\Lambda_{1}^{c}} g_{1}+f_{1} \cap_{\Lambda_{1}} g_{1}+f_{2} \cap_{\Lambda_{2}} g_{2}+f_{2} \cap_{\Lambda_{2}^{c}} g_{2} \\
=f_{1} \cap_{\Lambda_{1}^{c}} g_{1}+f_{2} \cap_{\Lambda_{2}^{c}} g_{2}=f \cap g,
\end{gathered}
$$


since the middle terms in (5) add up to 0 . This can be seen as follows: The restrictions $E_{\Delta_{i}} \longrightarrow E_{\Lambda_{i}}, i=1,2$, combine to a map

$$
E_{\Delta_{1}} \oplus E_{\Delta_{2}} \supset K \longrightarrow E_{\Lambda} \subset E_{\Lambda_{1}} \oplus E_{\Lambda_{2}}
$$

with image $A \otimes_{B} G_{\Phi}$. Denote $\hat{f}, \hat{g} \in E_{\Lambda}$ the respective images of the pairs $\left(f_{1}, f_{2}\right),\left(g_{1}, g_{2}\right) \in$ $K$. Then

$$
\hat{f} \cap \hat{g}=f_{1} \cap_{\Lambda_{1}} g_{1}+f_{2} \cap_{\Lambda_{2}} g_{2}
$$

So what we finally have to prove is that

$$
A \otimes_{B} G_{\Phi} \subset E_{\Lambda}
$$

is an isotropic subspace. For this we may even assume that $\Phi$ and thus also $\Lambda$ is simplicial (remember that $\Phi \cong \partial \Lambda_{i}$ ), otherwise take a simplicial refinement $\iota: \widehat{\Phi} \longrightarrow \Phi$ and a direct embedding $\mathcal{G} \longrightarrow \iota_{*}(\mathcal{A})$, where $\mathcal{A}$ is the structure sheaf of $\widehat{\Phi}$. There is an induced simplicial refinement $\widehat{\Lambda} \longrightarrow \Lambda$ and embedding $E_{\Lambda} \hookrightarrow A_{\widehat{\Lambda}}$ respecting the intersection product. Since $A \otimes_{B} G_{\Phi} \subset A \otimes_{B} A_{\widehat{\Phi}}$, our claim holds for $\Lambda$, if it does for $\widehat{\Lambda}$.

So let us now consider the case where $\Phi$ and hence also $\Lambda$ is simplicial. In that situation, it suffices to check that the evaluation map $\varepsilon: E_{\Lambda}=A_{\Lambda} \longrightarrow A[-2 n]$ vanishes on $A \otimes_{B} G_{\Phi}$. We use the formula (II) in section 4 .F for $\varepsilon$ :

$$
E_{\Lambda} \cong A_{\Lambda} \subset \bigoplus_{\sigma \in \Lambda^{n}} A_{\sigma} \longrightarrow Q(A), \quad f=\left(f_{\sigma}\right)_{\sigma \in \Lambda^{n}} \longmapsto \sum_{\sigma \in \Lambda^{n}} \frac{f_{\sigma}}{g_{\sigma}}
$$

But the $n$-cones in $\Lambda$ may be grouped in pairs $\sigma_{i} \in \Lambda_{i}, i=1,2$ with $\pi\left(\sigma_{1}\right)=\pi\left(\sigma_{2}\right)$. Then for $f \in A \otimes_{B} G_{\Phi}=A \otimes_{B} A_{\Phi}$, we have

$$
f_{\sigma_{1}}=f_{\sigma_{2}}, \text { while } g_{\sigma_{1}}=-g_{\sigma_{2}},
$$

and thus $\varepsilon(f)=0$. -

(D) The HR relations for $\boldsymbol{P}$. Since

$$
\bar{D} \cong I H^{*}\left(\Lambda_{i}, \partial \Lambda_{i}\right) \cong I H^{*-2}\left(\Lambda_{i}\right) \cong I H^{*-2}(\Phi)
$$

and the third module $E_{\Delta}$ in the exact sequence (44) is free, there is an associated exact sequence

$$
0 \longrightarrow I H^{*-2}(\Phi) \longrightarrow \bar{K} \longrightarrow I H(\Delta) \longrightarrow 0
$$

realizing $I H(\Delta)=\bar{E}_{\Delta}$ in the same way as before $E_{\Delta}$. Furthermore it is compatible with the natural Lefschetz operators on all three terms; we shall denote them simply $L$ in all cases. 
Now let $\zeta \in I H^{n-k}(\Delta), \zeta \neq 0$, be a primitive class, i.e., $L^{k+1}(\zeta)=0$. We can lift it to a pair $\xi=\left(\xi_{1}, \xi_{2}\right) \in \bar{K}$. We may actually assume that the classes $\xi_{i} \in I H^{n-k}\left(\Delta_{i}\right)$ are again primitive, i.e., $\xi_{i} \in I P^{n-k}\left(\Delta_{i}\right)$ : Because of $L^{k+1}(\zeta)=0$, we have

$$
L^{k+1}(\xi)=\eta \in I H^{n+k}(\Phi) \subset \bar{K}^{n+k+2},
$$

and since

$$
L^{k+1}: I H^{n-k-2}(\Phi)=I H^{(n-1)-(k+1)}(\Phi) \stackrel{\cong}{\longrightarrow} I H^{(n-1)+(k+1)}(\Phi)=I H^{n+k}(\Phi)
$$

is an isomorphism - by the assumption, HRR and thus HLT holds for the lower dimensional polytope $P_{1} \cap P_{2}-$, we may replace $\xi$ with $\xi-L^{-(k+1)}(\eta)$.

So now let both $\xi_{1}$ and $\xi_{2}$ be primitive. Since $\zeta \neq 0$, we have $\xi_{i} \neq 0$ for at least one index $i$. Thus

$$
(-1)^{(n-k) / 2} \zeta \cap L^{k}(\zeta)=(-1)^{(n-k) / 2} \xi_{1} \cap L^{k}\left(\xi_{1}\right)+(-1)^{(n-k) / 2} \xi_{2} \cap L^{k}\left(\xi_{2}\right)>0,
$$

since the HRR hold for $P_{1}$ and $P_{2}$.

\section{Deformation}

Proposition 6.1. The germ $G=G_{P}(F)$ of a normally trivial face $F \prec P$ can be deformed into the product $F \times \Pi(L)$, where $L:=L_{P}(F)$ denotes a link of $F$ in $P$.

Proof. We use the terminology of 3.5 and assume that $\stackrel{\circ}{F} \cap N=\{0\}$; so the affine span $U:=\operatorname{aff}(F)$ as well as $N$ are linear subspaces, and $V=U \oplus N$. Furthermore write $N=W \oplus \mathbf{R}$, such that $H=U \times W \times\{1\}$, and $L_{P}(F)=L \times\{1\}$ with a polytope $L \subset W$. Denote $\mathbf{u}_{1}, \ldots, \mathbf{u}_{r} \in U$ the vertices of $F$ and $\mathbf{w}_{1}, \ldots, \mathbf{w}_{s} \in W$ the vertices of $L$, then $G$ has vertices $\left(\mathbf{u}_{i}, 0,0\right)$ and $\left(\mathbf{u}_{i}+\mathbf{u}_{i j}, \mathbf{w}_{j}, 1\right)$ with suitable vectors $\mathbf{u}_{i j} \in U$.

Now let $G_{t}:=G \cap(U \times W \times[0, t])$ be the truncated germ, and consider on

$$
V=U \oplus N=U \oplus(W \oplus \mathbf{R})
$$

the linear isomorphism

$$
F_{t}:=\operatorname{id}_{U} \oplus t^{-1} \mathrm{id}_{N}
$$

Then $(0,1] \ni t \mapsto Q_{t}:=F_{t}\left(G_{t}\right)$ extends to a deformation $[0,1] \ni t \mapsto Q_{t}$ with $Q_{0}=$ $F \times \Pi(L)$. In fact the polytope $Q_{t}$ has vertices $\left(\mathbf{u}_{i}, 0,0\right)$ and $\left(\mathbf{u}_{i}+t \mathbf{u}_{i j}, \mathbf{w}_{j}, 1\right)$.

Theorem 6.2. Let $F \prec P$ be a normally trivial face of an $n$-dimensional polytope $P$, and assume that $F$ itself is a simple polytope. Then the HRR hold for $G=G_{P}(F)$ if they hold for lower dimensional polytopes. 
Proof. For $\operatorname{dim} F=0$, the germ $G=\Pi\left(L_{P}(F)\right)$ is a pyramid, so we may apply Proposition 5.1. Now let $\operatorname{dim} F>0$. Let us first give a

Survey of proof: We consider the deformation $Q_{t}, 0 \leq t \leq 1$, of Prop. 6.1, which deforms $Q_{1}=G$ into $Q_{0}=F \times \Pi(L)$. The HLT and the HRR hold on $Q_{0}$ according to the Künneth formula. Then we show that the HLT holds on $Q_{t}$ for all $t \in(0,1]$, cf. 6.4, hence the HR-forms on $I H\left(\Delta\left(Q_{t}\right)\right)$ are non-degenerate for any $t \in[0,1]$. Since the combinatorial type of the polytopes $Q_{t}$ is constant along the deformation, the Betti numbers are so too, in fact both $I H^{n-k}\left(\Delta\left(Q_{t}\right)\right), t \in[0,1]$ and the $k$-th HR-form $s_{k}^{t}$ on $I H^{n-k}\left(\Delta\left(Q_{t}\right)\right)$ depend continuously on $t \in[0,1]$, cf. 6.3. Since they are non-degenerate, their signature is independent of the parameter $t \in[0,1]$. Hence the HR-equations 1.2 hold for all $t$, since they do for $t=0$.

\section{(A) The deformation on the fan level:}

The case $t>0$ : For $t>0$ the fan $\Delta_{t}:=\Delta\left(Q_{t}\right)$ is "linearly equivalent" to $\Delta_{1}=\Delta(G)$, i.e., $\Delta_{t}$ is the image of $\Delta_{1}$ with respect to a linear isomorphism of the vector space $V^{*}$, namely the inverse $\left(F_{t}^{*}\right)^{-1}$ of the dual $F_{t}^{*}$ of the map $F_{t}: V \longrightarrow V$ transforming $G_{t}$ into $Q_{t}$ in the proof of 6.1. It provides an isomorphism

$$
F_{t}^{*}: \Delta_{t}=\Delta\left(Q_{t}\right) \longrightarrow \Delta\left(G_{t}\right)=\Delta(G)=\Delta\left(Q_{1}\right)=\Delta_{1} .
$$

Behaviour near 0: We replace the linear isomorphism $\left(F_{t}^{*}\right)^{-1}$ mapping $\Delta_{1}$ onto $\Delta_{t}$ with a $\Delta_{0}$-conewise-linear isomorphism $S_{t}: V^{*} \longrightarrow V^{*}$, such that

$$
S_{t}\left(\Delta_{0}\right)=\Delta_{t}
$$

The construction of $S_{t}$ is as follows: Consider the subfan $\Gamma \preceq \Delta(G)$ generated by the cones $\sigma\left(F_{0}\right)$, where $F_{0} \preceq G$ is a minimal face projecting onto the entire pyramid $\Pi(L)$, i.e., $\pi\left(F_{0}\right)=\Pi(L)$ with the projection $\pi: V=U \oplus N \longrightarrow N$. The support $|\Gamma|$ is the graph of a map $H: U^{*} \longrightarrow N^{*}$. In fact that map is $\Phi$-conewise linear for the outer normal fan $\Phi:=\Delta(F)$ of the polytope $F \subset U$, and

$$
S_{t}: U^{*} \oplus N^{*} \longrightarrow U^{*} \oplus N^{*},(\mathbf{x}, \mathbf{y}) \mapsto(\mathbf{x}, \mathbf{y}+t H(\mathbf{x}))
$$

then defines a $\Delta_{0}$-conewise linear isomorphism with the desired properties. Note here that $\Delta_{0}$ is the product

$$
\Delta_{0}=\Phi \times \Lambda
$$

of the (simplicial) fan $\Phi:=\Delta(F)$ in $U^{*}$ and the fan $\Lambda:=\Delta(\Pi(L))$ in $N^{*}$.

(B) Pull back isomorphisms: Both $\left(F_{t}^{*}\right)^{-1}$ and $S_{t}$ act on the global sections of the structure resp. the intersection cohomology sheaf by pull back. Let us write

$$
A_{t}:=A_{\Delta_{t}}, E_{t}:=E_{\Delta_{t}} .
$$


Then $\left(F_{t}^{*}\right)^{-1}$ induces $A$-module isomorphisms

$$
A_{t} \longrightarrow A_{1}, E_{t} \longrightarrow E_{1}
$$

in particular $I H\left(\Delta_{t}\right) \cong I H\left(\Delta_{1}\right)$ in a natural way, while for $S_{t}$ the corresponding maps

$$
A_{t} \longrightarrow A_{0}, E_{t} \longrightarrow E_{0}
$$

both denoted $S_{t}^{*}$, are only isomorphisms of graded vector spaces due to the fact that for the subalgebra $A \subset A_{t}$ of "global polynomials" we in general have $S_{t}^{*}(A) \not \subset A \subset A_{0}$. So we can not any longer identify $I H\left(\Delta_{t}\right)$ in a reasonable way with $I H\left(\Delta_{0}\right)$.

(C) Continuity statements: That everything is continuous in $t \in(0,1]$ follows now immediately from the fact that the strictly convex function on $\Delta_{1}=\Delta(G)$ given by the vertices of $G_{t}$ is continuous in $t$. Near 0 there is no natural trivialization of the family $I H\left(\Delta_{t}\right)$; instead we have to represent $I H\left(\Delta_{t}\right)$ as a factor space $E / M_{t}$ of a bigger vector space $E$ independent of $t$ with varying subspace $M_{t}$ :

Proposition 6.3. There is a finite dimensional graded vector space $E$ and continuous families of

1. subspaces $M_{t} \subset E$ of constant dimension, such that in a natural way

$$
I H\left(\Delta_{t}\right) \cong E / M_{t}
$$

2. endomorphisms $\hat{L}_{t}: E \longrightarrow E$ with $\hat{L}_{t}\left(M_{t}\right) \subset M_{t}$ inducing the Lefschetz operator of $\Delta_{t}=\Delta\left(Q_{t}\right)$

3. symmetric bilinear forms $\beta_{t}: E \times E \longrightarrow \mathbf{R}$ with $\beta_{t}\left(M_{t}, E\right)=0$, inducing the intersection product.

Proof. Let us start with

The vector spaces $E$ and $M_{t}$ : Write $\Delta:=\Delta_{0}$. We take:

$$
E:=\bigoplus_{q=0}^{2 n} E_{0}^{q}
$$

and

$$
M_{t}:=S_{t}^{*}\left(\mathfrak{m} E_{t}\right) \cap E \subset E .
$$

The subspaces $M_{t}$ can be represented in the form $\Phi_{t}\left(\mathfrak{m}^{<2 n} \otimes E\right)$ with the continuous family of linear maps

$$
\Phi_{t}: \mathfrak{m}^{<2 n} \otimes E \longrightarrow E, g \otimes f \mapsto S_{t}^{*}(g) f
$$


furthermore, since $\Delta_{t}$ and $\Delta$ are combinatorially equivalent, we get that $\operatorname{dim} M_{t}=\operatorname{dim} E-$ $\operatorname{dim} I H\left(\Delta_{t}\right)$ is independent of $t$. The map $\hat{L}_{t}$ is multiplication with $S_{t}^{*}\left(\psi_{t}\right):=\psi_{t} \circ S_{t}$, except on the highest weight subspace $E_{\Delta}^{2 n}$, where it vanishes. Here $\psi_{t}$ denotes the strictly convex function belonging to $Q_{t}$.

Continuity of the intersection product: Here we consider in general the situation, where we have a fan $\Delta$ in $V^{*}$ and $\Delta_{t}:=S_{t}(\Delta)$ with a continuous family of $\Delta$-conewise linear isomorphisms $S_{t}: V^{*} \longrightarrow V^{*}$. The bilinear form we consider is

$$
\beta_{t}: E \times E \subset E_{\Delta} \times E_{\Delta} \stackrel{\left(S_{t}^{-1}\right)^{*}}{\longrightarrow} E_{t} \times E_{t} \stackrel{\cap}{\longrightarrow} A[-2 n] \longrightarrow A^{0} \cong \mathbf{R}
$$

where the last arrow is (up to the shift) the projection $A=A^{0} \oplus \mathfrak{m} \longrightarrow A^{0}$.

Let us first look at the case of a

Simplicial fan $\Delta$ : Then we have $\mathcal{E}=\mathcal{A}$. Take $r, s$ with $r+s=2 n$. We have to show that the map

$$
A_{0}^{r} \times A_{0}^{s} \stackrel{\left(S_{t}^{-1}\right)^{*}}{\longrightarrow} A_{t}^{r} \times A_{t}^{s} \stackrel{\cap}{\longrightarrow} A^{0}=\mathbf{R}
$$

depends continuously on $t \in[0,1]$. But that map may be rewritten as

$$
A_{0}^{r} \times A_{0}^{s} \stackrel{\text { mult }}{\longrightarrow} A_{0}^{2 n} \stackrel{\left(S_{t}^{-1}\right)^{*}}{\longrightarrow} A_{t}^{2 n} \stackrel{\varepsilon_{t}}{\longrightarrow} A^{0}=\mathbf{R},
$$

using the fact that $\left(S_{t}^{-1}\right)^{*}$ commutes with the multiplication of functions. Here $\varepsilon_{t}$ denotes the restriction of the evaluation map

$$
A_{t} \longrightarrow A[-2 n]
$$

to the $2 n$-th weight space $A_{t}^{2 n}$. It is well defined after having fixed a volume form on $V$. So, eventually we have to check that the map

$$
A_{0}^{2 n} \stackrel{\left(S_{t}^{-1}\right)^{*}}{\longrightarrow} A_{t}^{2 n} \stackrel{\varepsilon_{t}}{\longrightarrow} \mathbf{R}
$$

depends continuously on $t \in[0,1]$. Take any $n$-cone $\sigma_{0} \in \Delta=\Delta_{0}$, set $\sigma_{t}:=S_{t}\left(\sigma_{0}\right) \in \Delta_{t}$ and choose a non-negative function $f_{t} \in A_{\left(\sigma_{t}, \partial \sigma_{t}\right)}^{2 n} \subset A_{t}^{2 n}$, the product of linear forms $\in\left(V^{*}\right)^{*}$, whose $\wedge$-product is up to sign the volume form on $V$. Denote $T_{t}: V^{*} \longrightarrow V^{*}$ the linear map, which coincides with $S_{t}$ on $\sigma_{0}$. Then we have $S_{t}^{*}\left(f_{t}\right)=\operatorname{det}\left(T_{t}\right) f_{0}$, and the map $\varepsilon_{t} \circ\left(S_{t}^{-1}\right)^{*}: A_{0}^{2 n} \longrightarrow \mathbf{R}$ can be thought of as $\operatorname{det}\left(T_{t}\right)^{-1}$ times the projection operator $A_{0}^{2 n} \longrightarrow \mathbf{R} f_{0} \subset A_{0}^{2 n}$ with kernel $M_{t}^{2 n} \subset E^{2 n}=A_{0}^{2 n}$, since $\varepsilon_{t}\left(f_{t}\right)=1$. That yields the desired continuity with respect to $t \in[0,1]$.

The general case: Take a simplicial refinement $\Sigma \stackrel{\iota}{\longrightarrow} \Delta$ and consider an enbedding

$$
\mathcal{E} \hookrightarrow \iota_{*}(\mathcal{A}) \text { and } E_{\Delta} \hookrightarrow A_{\Sigma}
$$


as in section 4.F. For $\Sigma_{t}:=S_{t}(\Sigma)$ it induces embeddings

$$
E_{t}:=E_{\Delta_{t}} \hookrightarrow A_{t}:=A_{\Sigma_{t}}
$$

which according to $\mathrm{BBFK}_{3}$ respect the intersection pairings. Then the intersection product takes the form

$$
E_{0}^{r} \times E_{0}^{s} \longrightarrow A_{0}^{r} \times A_{0}^{s} \stackrel{\left(S_{t}^{-1}\right)^{*}}{\longrightarrow} A_{t}^{r} \times A_{t}^{s} \stackrel{\cap}{\longrightarrow} A^{0}=\mathbf{R},
$$

so the simplicial case applies.

(D) Statement HLT for the $Q_{t}, t \in(0,1]$ : Finally we show

Proposition 6.4. The HLT holds for the polytopes $Q_{t}, t \in(0,1]$, in particular the HRforms are non-degenerate for all $t \in[0,1]$.

Proof. Since the linear isomorphism $F_{t}^{*}: V^{*} \longrightarrow V^{*}$ induces an isomorphism

$$
I H\left(\Delta_{t}\right) \stackrel{\cong}{\longrightarrow} I H\left(\Delta_{1}\right)
$$

we may replace $\Delta_{t}$ with $\Delta:=\Delta_{1}=\Delta(G)=\Delta\left(G_{t}\right)$. Denote $\psi:=\psi_{t}$ the function given by the vertices of $G_{t}$ and $L=L_{t}$ the corresponding Lefschetz operator. Because of Poincaré duality it suffices to prove that the corresponding $k$-th iteration of the Lefschetz map $L^{k}: I H^{n-k}(\Delta) \longrightarrow I H^{n+k}(\Delta)$ is injective for $k>0$. We have

$$
\Delta=\Theta \cup \Theta_{0}
$$

with the subfan $\Theta=\operatorname{st}(\sigma(F))$ corresponding to the ridge $F \preceq G$ of the "hip roof" $G$, and the subfan $\Theta_{0}:=\operatorname{st}(\nu(G \cap H))$ associated to the bottom or cut facet $G \cap H$. The rays of $\Delta$ not contained in $\sigma(F)$ are the outer normal rays $\varrho_{i}:=\nu\left(F_{i}\right)$ of the facets $F_{0}, \ldots, F_{r}$ of $G$ not containing $F$ - say, $F_{0}:=G \cap H \prec G$ is the bottom of $G$. Since $F$ is simple, they are normally trivial in $G$ (To see that use the fact that $G$ is combinatorially equivalent to $F \times \Pi(L)$, cf. 3.5] and that the $F_{1}, \ldots, F_{r}$ under that equivalence correspond to facets of $F$ times the pyramid $\Pi(L)$, while for the cut facet $F_{0}$ the claim is obvious), in particular we find that any $n$-cone $\sigma \succeq \varrho_{i}$ is the sum of $\varrho_{i}=\nu\left(F_{i}\right)$ and the unique opposite facet of $\sigma$ (corresponding to the unique edge starting in the vertex the cone $\sigma$ is associated with, and not contained in the facet $F_{i}$.)

As a consequence, there are (unique) functions $\psi_{i} \in A_{\Delta}^{2}$ vanishing outside $\Theta_{i}:=$ $\operatorname{st}\left(\nu\left(F_{i}\right)\right)$ with $\psi_{i}=\psi$ on the ray $\nu\left(F_{i}\right)$ for $i=0, \ldots, r$. On the other hand, we may assume $0 \in F$ resp. $\left.\psi\right|_{\sigma(F)}=0$. So altogether we have

$$
\psi=\sum_{i=0}^{r} \psi_{i} .
$$


Now assume $k \equiv n \bmod (2), 0<k \leq n$ and $\xi \in I H^{n-k}(\Delta)$ with $L^{k}(\xi)=0$. For $\Theta_{i}:=$ $\operatorname{st}\left(\nu\left(F_{i}\right)\right)$ we show $0=\left.\xi\right|_{\Theta_{i}} \in I H\left(\Theta_{i}\right)$. First of all $L^{k}(\xi)=\psi^{k} \xi=0$ gives

$$
0=\xi \cap \psi^{k} \xi=\sum_{i=0}^{r} \xi \cap \psi_{i} \psi^{k-1} \xi=\sum_{i=0}^{r} \xi_{i} \cap \varphi_{i}^{k-1} \xi_{i}
$$

with $\xi_{i}:=\left.\xi\right|_{\Theta_{i}} \in I H^{n-k}\left(\Theta_{i}\right) \cong I H^{(n-1)-(k-1)}\left(\Delta\left(F_{i}\right)\right)$, the strictly convex conewise linear function $\varphi_{i} \in A_{\Delta\left(F_{i}\right)}^{2}$ being the pullback of $\left.\psi\right|_{\partial \Theta_{i}}$ with respect to the inverse of $\left.\pi\right|_{\partial \Theta_{i}}$ : $\partial \Theta_{i} \stackrel{\cong}{\longrightarrow} \Delta\left(F_{i}\right)$, where $\pi$ is the quotient projection $\pi: V^{*} \longrightarrow V^{*} / \operatorname{span}\left(\nu\left(F_{i}\right)\right)$, the intersection product $\xi_{i} \cap \varphi_{i}^{k-1} \xi_{i}$ referring to $\Delta\left(F_{i}\right)$. The equality $\xi \cap \psi_{i} \psi^{k-1} \xi=\xi_{i} \cap \varphi_{i}^{k-1} \xi_{i}$ is obtained as follows: If we use the internal product approach to the intersection product, we obtain a commutative diagram

$$
\begin{array}{ccc}
\left(\xi_{i}, \xi_{i}\right) & & \left.\left(\xi, \psi_{i} \xi\right)\right|_{\Theta_{i}} \\
\in & & \in \\
I H^{(n-1)-(k-1)}\left(\Delta\left(F_{i}\right)\right) \times I H^{(n-1)-(k-1)}\left(\Delta\left(F_{i}\right)\right) & \longrightarrow & I H^{n-k}\left(\Theta_{i}\right) \times I H^{n-k+2}\left(\Theta_{i}, \partial \Theta_{i}\right), \\
\downarrow & & \downarrow \\
I H^{2 n-2}\left(\Delta\left(F_{i}\right)\right) & \stackrel{\cong}{\longrightarrow} & I H^{2 n}\left(\Theta_{i}, \partial \Theta_{i}\right)
\end{array}
$$

where the left vertical arrow denotes the $(k-1)$ rst HR-form of $\Delta\left(F_{i}\right)$ and the right one is the intersection product composed in one argument with multiplication with $\psi^{k-1}$. The second component of the upper horizontal homomorphism and the lower one are pull back to the fan $\Theta_{i}$ followed by the Thom isomorphism (cf. the proof of [5.1), i.e., multiplication with $\psi_{i}$.

Now as a consequence of $L^{k}(\xi)=0$ we have $\xi_{i} \in I P^{(n-1)-(k-1)}\left(\Delta\left(F_{i}\right)\right)$. Hence the HRR for $\Delta\left(F_{i}\right)$ give that all the summands are either non-negative or non-positive. So necessarily $\xi_{i} \cap \varphi_{i}^{k-1} \xi_{i}=0$ resp. $\xi_{i}=0$ for $i=0, \ldots, r$. In particular $\xi_{0}=0$ and the exact sequence

$$
0 \longrightarrow I H^{n-k}(\Theta, \partial \Theta) \longrightarrow I H^{n-k}(\Delta) \longrightarrow I H^{n-k}\left(\Theta_{0}\right) \longrightarrow 0
$$

tells us that $\xi \in I H^{n-k}(\Theta, \partial \Theta) \subset I H^{n-k}(\Delta)$, and thus, in order to conclude $\xi=0$, it suffices to prove that $\left.L^{k}\right|_{I H^{n-k}(\Theta, \partial \Theta)}$ is injective for $k>0$ resp. that dually $L^{k}$ : $I H^{n-k}(\Theta) \longrightarrow I H^{n+k}(\Theta)$ is surjective for $k>0$. In fact we show, that the graded vector space $I H(\Theta) / L^{k}(I H(\Theta))$ has weights at most $n+k-1$.

Now the fan $\Theta$ has the form

$$
\Theta=S(\Phi \times \sigma(0)) \subset U^{*} \oplus N^{*}
$$

with the cone $\sigma(0) \subset N^{*}$ associated to the apex $0 \in \Pi(L)$ of the pyramid $\Pi(L) \subset N$ and $S:=S_{1}$. Using the induced vector space isomorphism

$$
S^{*}: E_{\Theta} \longrightarrow E_{\Phi \times \sigma(0)} \cong A_{\Phi} \otimes E_{\sigma(0)}
$$


we can write

$$
\left.I H(\Theta) \cong A_{\Phi} \otimes E_{\sigma(0)} / S^{*}(\mathfrak{m})\left(A_{\Phi} \otimes E_{\sigma(0)}\right)\right)
$$

with $S^{*}(\mathfrak{m}) \subset A_{\Phi \times \sigma(0)}$. Since $\left.\psi\right|_{\sigma(F)}=0$ (because of $0 \in F$ ) and $\Theta=\operatorname{st}(\sigma(F)$ ) (remember that $\left.\sigma(F)=o \times \sigma(0) \subset U^{*} \oplus N^{*}\right)$, we can write $\left.\psi\right|_{\Theta}=\chi \circ p$ with the projection $p: U^{*} \oplus N^{*} \longrightarrow U^{*}$ and a function $\chi \in A_{\Phi}^{2}$. Now in order to compute $I H(\Theta) / L^{k}(I H(\Theta))$ we have to regard on $A_{\Phi} \otimes E_{\sigma(0)}$ the "twisted" $A$-module structure obtained from that of $E_{\Theta}$ by pull back via $S$, with other words, a function $f \in A$ acts on $A_{\Phi} \otimes E_{\sigma(0)}$ by "standard" multiplication with $f \circ S \in A_{\Phi \times \sigma(0)}$.

Now write $A=C \otimes D$ with the polynomial algebras $C:=S\left(\left(U^{*}\right)^{*}\right)$ resp. $D:=$ $S\left(\left(N^{*}\right)^{*}\right)$ on $U^{*}$ resp. $N^{*}$. Then $g=g(\mathbf{x}) \in C$ acts on the first factor $A_{\Phi}$ only, while $h \in D$ acts by standard multiplication with $h(\mathbf{y}+H(\mathbf{x}))$. We have now to divide by the submodule obtained by multiplication with $\mathfrak{m}_{C}, \chi^{k}, \mathfrak{m}_{D}$. Looking first at $\mathfrak{m}_{C}$ and $\chi^{k}$ gives

$$
\left(I H(\Phi) / L^{k}(I H(\Phi)) \otimes E_{\sigma(0)},\right.
$$

a $D$-module. The graded vector space $F:=I H(\Phi) / L^{k}(I H(\Phi))$ has weights at most $s+k-1, s:=\operatorname{dim} U^{*}<n$, according to the HLT for $F$. It admits a descending filtration by the $D$-submodules

$$
F^{\geq i} \otimes E_{\sigma(0)}, 0 \leq i \leq s+k
$$

with free successive quotients

$$
F^{\geq i} \otimes E_{\sigma(0)} / F^{\geq i+1} \otimes E_{\sigma(0)} \cong\left(F^{\geq i} / F^{\geq i+1}\right) \otimes E_{\sigma(0)}
$$

since $h \in D$ acts only on the second factor of the right hand side - the twist being factored out. The short exact sequences

$$
0 \longrightarrow F^{\geq i+1} \otimes E_{\sigma(0)} \longrightarrow F^{\geq i} \otimes E_{\sigma(0)} \longrightarrow F^{\geq i} \otimes E_{\sigma(0)} / F^{\geq i+1} \otimes E_{\sigma(0)} \longrightarrow 0
$$

remain exact after reduction $\bmod \mathfrak{m}_{D}$ : The third terms being free $D$-modules, they are split. So, since the third non-trivial term has weights $<i+t$ with $t:=\operatorname{dim} N^{*}$ according to $\mathrm{BBFK}_{2}$ 1.7, we see by descending induction on $i$ that the reduction of $F^{\geq i} \otimes E_{\sigma(0)}$ has weights at most $(s+k-1)+t=n+k-1$. The case $i=0$ gives the claim.

This finishes the proof of both, 6.4 and 6.2

\section{References}

[BBFK 1$]$ G. Barthel, J.-P. Brasselet, K.-H. Fieseler And L. Kaup, Equivariant Intersection Cohomology of Toric Varieties, Algebraic Geometry, Hirzebruch 70, 45-68, Contemp. Math. 241, Amer. Math. Soc., Providence, R.I., 1999. 
[BBFK 2$]$ G. Barthel, J.-P. Brasselet, K.-H. Fieseler and L. Kaup, Combinatorial Intersection Cohomology for Fans, Tôhoku Math. J. 54 (2002), 1-41.

[BBFK 3$]$ G. Barthel, J.-P. Brasselet, K.-H. Fieseler and L. Kaup, Combinatorial Duality and Intersection Product: A Direct Approach, (pr)e-print math.AG/0309352v1 (21 pages), 2003, to appear in Tôhoku Math. J.

[BreLu 1 P. BREssler AND V. Lunts, Intersection cohomology on nonrational polytopes, Compos. Math. 135 (2003), 245-278.

$\left[\mathrm{BreLu}_{2}\right]$ P. BREssler AND V. LunTs, Hard Lefschetz theorem and Hodge-Riemann relations for intersection cohomology of nonrational polytopes, (pr)e-print math.AG/0302236 v2 (46 pages), 2003.

[Bri] M. Brion, The structure of the polytope algebra, Tôhoku Math. J. 49 (1997), 1-32.

[Ka] K. Karu, Hard Lefschetz Theorem for Nonrational Polytopes, Invent. Math. 157 (2004), 419-447.

[Mc] P. McMullen, On simple Polytopes, Invent. Math. 113 (1993), 419-444.

[St] R. Stanley, Generalized h-vectors, intersection cohomology of toric varieties and related results, M. Nagata, H. Matsumura, eds., Commutative Algebra and Combinatorics, 187213, Adv. Stud. Pure Math. 11, Kinokunia, Tokyo, and North Holland, Amsterdam/New York, 1987.

[Ti] V. A. Timorin, An analogue of the Hodge-Riemann relations for simple convex polytopes, Russian Mathematical Surveys 54.2 (1999), 381-426

G. Barthel, L. Kaup

Fachbereich Mathematik und Statistik

Universität Konstanz Fach D 203

D-78457 Konstanz

Germany

E-mail adresses: Gottfried.Barthel@uni-konstanz.de, Ludger . Kaup@uni-konstanz.de

J.P. BRASSELET

IML/CNRS, Luminy CASE 907

F-13288 Marseille Cedex 9

FRANCE

E-mail adress: jpb@iml.univ-mrs.fr 
K.-H. FiESELER

Matematiska Institutionen Box 480

UPPSALA UNIVERSITET

SE-75106 UPPSALA

SWEDEN

E-mail adress: khf@math.uu.se 